\title{
L'impeccabilité du Prophète Muḥammad dans le credo sunnite
}

D'al-Aš́arī (m. 324/935) à Ibn Taymiyya (m. 728/1328)

The Sinlessness of the Prophet Muhammad in Sunni Thought

$$
\text { عصمة الرسول محمد في عقيدة أهل السنة }
$$

\section{Nadjet Zouggar}

\section{(2) OpenEdition}

\section{Journals}

Édition électronique

URL : http://journals.openedition.org/beo/296

DOI : $10.4000 /$ beo.296

ISBN : 978-2-35159-335-6

ISSN : 2077-4079

Éditeur

Presses de l'Institut français du Proche-Orient

Édition imprimée

Date de publication : 1 mai 2012

Pagination : 73-89

ISBN : 978-2-35159-193-2

ISSN : 0253-1623

Référence électronique

Nadjet Zouggar, "L'impeccabilité du Prophète Muhammad dans le credo sunnite », Bulletin d'études orientales [En ligne], Tome LX I mai 2012, mis en ligne le 31 mai 2012, consulté le 19 avril 2019. URL: http://journals.openedition.org/beo/296 ; DOI : 10.4000/beo.296 


\title{
L'IMPECCABILITÉ DU PROPHÈTE MUHAMMAD DANS LE CREDO SUNNITE D’al-Aš́arī (m. 324/935) à Ibn Taymiyya (m. 728/1328)
}

\author{
Nadjet ZOUGGAR \\ Université de Toulouse Le Mirail
}

Dans la littérature religieuse de l'islam, le substantif 'ișma ${ }^{1}$ renvoie à plusieurs sens qui convergent vers l'idée de « protection » ou de « préservation » de l'erreur ou du péché. Le mot 'ișma englobe ainsi l'idée d'infaillibilité, entendue comme la capacité d'être à l'abri de l'erreur, qu'elle soit produite en conscience ou non ; ce qui inclut l'erreur d'estimation (tark al-awlā) ou bien l'erreur dans l'effort d'interprétation (al-iğtihād), le lapsus (al-zalal) et l'inadvertance (al-sahw). La notion couvre également le sens d'impeccabilité, c'est-à-dire

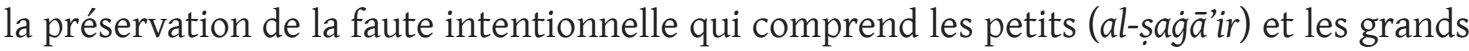
péchés (al-kabā’ir).

Si la tendance à blanchir le Prophète Muhammad en l'exemptant de toute erreur est manifeste dès les premières écritures biographiques de l'islam ${ }^{2}$, il est important de noter que le Coran et le Hadith ne mentionnent pas la iṣma des prophètes entendue comme une préservation de l'erreur ou bien du péché.Les sources scripturaires d'autorité de l'islam ne permettent donc pas d'établir la doctrine de l'impeccabilité ni même celle de l'infaillibilité des prophètes ${ }^{3}$. Cette notion est probablement entrée dans l'islam par la pensée chiite, sous l'influence de croyances venues de l'Orient ancien, assignant aux hommes investis

1. Le mot 'ișma est une déclinaison de la racine '-ș-m. Al-ișma: al-man' (interdiction) ; 'așamahu: waqāhu (protection) ; al-ḥifz (la préservation) ; al-itișām bi-šay': al-imtisāk bi-šay' (l'idée de s'attacher ou de s'en tenir à quelque chose). Voir IBn Manẓur, Lisān al-'arab, vol. 10, p. 175-178.

2. Notamment chez Ibn Sa'd, Ibn Hišām et al-Ṭabarī. Le hadīt de Muhammad purifié par des anges alors qu'il était enfant illustre ce phénomène : «On m'envoya deux hommes vêtus de blanc avec une écuelle en or pleine de neige ; ils m'ouvrirent la poitrine, me fendirent le cœur afin d'en extraire une adhérence noire, ils me lavèrent ensuite le cœur et la poitrine avec de la neige » (Iвn Hı̌̌ĀM, Sīrat al-nabī, p. 176).

3. Le Coran mentionne toutefois des dérivés de la même racine, notamment: «wa-'tașimū bi-ḥabli l-Lâhi ğamîan » (mettez-vous hors de péril en vous rattachant à la protection de Dieu, Coran 3, 103) et «wa-Allāh ya'șimuka min al-nās» (Et Dieu te mettra hors d'atteinte des hommes, Coran 5, 67). Seul un hadìt cité dans le recueil d'Ibn Hanbal évoque la 'ișma dans un sens qui se rapproche de l'idée d'impeccabilité : «Si vous adoptez mon exemple, car je suis votre Prophète, je n’en serai digne si je n'étais préservé (ma ș̣ūm) de Satan ». Voir Wensinck 1965, vol. 4, p. $249-250$. 
par une instance divine, afin de guider leur communauté, nombre d'attributs surnaturels ${ }^{4}$. Les mu'tazilites avaient adopté la doctrine de l'impeccabilité morale des prophètes pour l'ériger en argument rationnel dans leur démonstration de la validité du prophétisme (dalā’l al-nubuwwa). Par la suite, la doctrine fut naturellement absorbée par le credo sunnite avec la formation des traités de kalām mātūridite et ašarite. On la voit apparaître dans les professions de foi sous forme d'affirmation et dans les questions traditionnelles (sam 'iyyāt) des traités de kalām où elle est devenue incontournable ${ }^{5}$.

Toutes ces écoles divergeaient quant à la portée et la nature de la ișma. Elle relevait selon les mu'tazilites et les chiites du même argument rationnel qui justifie l'envoi de prophètes, à savoir une bienveillance obligatoire pour Dieu (luțf wäğib) envers ses créatures. Les aša arites, eux, invoquaient le plus souvent la tradition ex auditu (al-sam) et plus précisément, le consensus des savants $(i g ̆ m \bar{a})$ pour parler de cette notion ${ }^{6}$. Dans un premier temps, ces derniers semblent avoir restreint l'utilité de la 'ișma à la sauvegarde de l'intégrité de la révélation, ne visant alors que l'infaillibilité du Prophète dans la transmission du message divin. Mais en insistant sur la période qui suit la révélation, l'impeccabilité du Prophète entendue comme une inhérence absolue allait pourtant faire son chemin. C'est ainsi que chez les ašarites tardifs, la doctrine finit par englober les deux sens d'impeccabilité et d'infaillibilité totales de tous les prophètes, à partir de leur investiture.

Partisans d'une lecture littéraliste de la révélation, les traditionnistes que l'hérésiologie ašarite surnommait «hašwiyya » sont restés attachés au sens apparent des versets coraniques où les prophètes sont admonestés et n'ont donc pas adhéré à la doctrine qui nous intéresse ici ${ }^{7}$. Selon eux, ces versets témoignent explicitement de ce que tous les prophètes, y compris Muhammad, ont commis des péchés avant et pendant leur mission ${ }^{8}$.

\section{AL-AŠ`ARĪ ET SES PREMIERS DISCIPLES : UNE IMPECCABILITÉ LIMITÉE}

Aucun des ouvrages d'al-Ašsarī (m.324/935) qui nous sont parvenus ne permet d'affirmer qui'il adhérait à la doctrine de l'impeccabilité ou de l'infaillibilité des prophètes 9 .

4. Pour des éclaircissements sur ce point, il faut se reporter aux études du courant «comparatiste » entre le monothéisme biblique et les religions de l'Orient ancien qui font ressortir les origines de nombreuses croyances comme celle qui nous occupe ici. Voir SFAR 1998, p. 157-197.

5. WENSINCK 1965, p. 217-218.

6. Al-BAĠDĀDĪ, Ușūl, p. 167.

7. IBN BAȚȚ, al-Šarh, p. 120.

8. Nous évoquons plus bas les principaux passages du Coran invoqués contre la ișma.

9. En effet, sa célèbre profession de foi, al-Ibāna, ne mentionne pas la 'ịsma. Son traité d'hérésiologie Maqālat expose, avec une neutralité remarquable, les opinions des murği ites, des rāfiḍites et des mu'tazilites sur la question, sans mention aucune de l'opinion des siens (voir AL-Ašc ARĪ, Maqālāt, p. 48-49, p. 151, p. 226-227). Et le Kitāb al-luma n'apporte rien de plus. Ce mutisme a d'ailleurs laissé libre cours à des interprétations divergentes dans les études d'islamologie. Dans l'article « 'iṣma » de l'Encyclopédie de l'Islam, Madelung (1971, p. 191) écrivait : « L’opinion attribuée plus tard à al-Ašarī, selon laquelle les prophètes sont exempts de l'erreur et du péché après mais non avant leur mission, n'est probablement pas authentique, elle reflète cependant la doctrine postérieure généralement professée 
Aussi, lorsqu'on considère le parcours de ses idées, les disciples de ce fondateur d'un kalām consensuel ont eu à le suivre dans sa démarche de conciliation entre, d'une part, les traditionnistes représentés par une école hanbalite très vigoureuse à son époque et, de l'autre, le courant mu'tazilite dont il est resté tributaire même après son départ de leur école ${ }^{10}$.

Pour autant, si les sources directes d'al-Aš́arī ne nous renseignent pas sur son attitude vis-à-vis de la 'ị̣ma des prophètes, le Muğarrad maqālāt al-Aš́arī, qui est une synthèse de sa doctrine établie par son disciple Abū Bakr Ibn Fūrak (m. 406/1015), procure quelques éléments éclairants.

Nous y apprenons d'abord qu'al-Aš́arī enseignait qu'il existe des gens qualifiés par la 'ișma ( $m a$ ș̣ūmūn) en dehors des prophètes ${ }^{11}$. Sa théorie énonce en substance que si Dieu accorde ses grâces (alțâf), dont fait partie la 'ișma, à une personne assujettie (mukallaf) sans qu'un grand péché vienne obscurcir le tableau, on peut alors la considérer comme préservée dans l'absolu (mașūm muțlaqan). Et si cette personne témoigne de l'unicité de Dieu (tawhīd) et s'en remet à Lui pour la résurrection, elle est dite "préservée de la mécréance » (ma șūm min al-kufr). Ibn Fūrak transmet à cet effet : « Il [al-Ašcarī] disait qu'il ne niait pas qu'il puisse y avoir des gens dont Dieu sait qu'il ne commettront pas de grands péchés, en dehors des envoyés ; ce sont là des "préservés dans l'absolu" ». Mais notre rapporteur d'objecter aussitôt :

"Nous ne professons pas cela pour ce qui concerne un individu en dehors des prophètes et envoyés (lā yuqāl ḍ̂ālika 'inda-nā fi wāhid bi-'ayni-hi mā halā al-anbiyā' wa-lmursalin) sauf ce qui est transmis par la communauté lorsqu'elle s'accorde sur un cas qui est validé, car le consensus est préservé de l'erreur ${ }^{12}$. »

Le commentateur se démarque ici de la doctrine de son maître qui semble relativiser le caractère exclusif de la 'ișma en l'admettant de fait pour toute personne croyante qui n'a pas commis de grand péché. Al-Aš́arī aurait donc opté pour un sens pratique de la notion de 'ișma, en rupture avec l'idée d'un privilège divin accordé aux prophètes afin qu'ils guident mieux leurs communautés. Une idée largement répandue chez les mu'tazilites et les chiites dont certains de ses disciples tardifs se feront l'écho.

par les ašcarites qui limitaient l'exemption à la période postérieure à la mission et admettaient, pour la période antérieure, les fautes graves et légères, mais non l'incroyance ». Et dans son ouvrage sur la doctrine d'al-Ašcarī, D. Gimaret (1990, p. 459) s'opposant à l'avis de W. Madelung déclarait : «Je ne vois pas ce qui permet à Madelung d'affirmer que l'attribution à al-Aš'arī d'une telle thèse "n'est probablement pas authentique". Les indications de Pazdāwī à ce sujet sont en tout cas entièrement confirmées par le Muğarrad. Et qu'Aš́arī ait pensé ainsi n'a, en soi, rien de surprenant ».

10. Al-Ašarī connaissait certainement l'opinion de son maître Abū-'Alī al-Ǧubba'î (m. 303/915-916) sur la 'ișma, une opinion rapportée plus tard par les théologiens ašarites en ces termes : « Il est inadmissible que les prophètes commettent intentionnellement un péché, petit ou grand soit-il, mais il est possible qu'ils commettent de petites erreurs dans leurs efforts d'interprétation ». Voir AL-BAǴdĀDĪ, Ușūl, p. 168 ; AL-RĀZī, al-Mahșūul, vol I, p. 501 ; AL-İ̄̄̄i, al-Mawāqif, p. 265.

11. Al-Ǎ̌́ ARĪ, Muğarrad, p. 109.

12. Al-Aš́ ARĪ, Muğarrad, p. 126. 
Plus loin, le problème d'une préservation du péché avant, de même que pendant la prophétie, est évoqué en ces termes :

« [Al-Ašcarī] dit que le péché et l'erreur étaient possibles chez eux avant la révélation, quant à l'après, nous n'avons pas trouvé de texte de lui attestant cela. C'est pourquoi il interprétait "Adam désobéit à son seigneur et s'égara", comme étant [valable] pour ce qui est antérieur à la prophétie. Car Dieu en a fait un prophète après l'avoir chassé du paradis ${ }^{13} \cdot »$

Ibn Fūrak rapporte un dernier raisonnement attribué à son maître : si les théologiens admettent ( $a g ̌ a \bar{z} \bar{u})$ que les prophètes puissent commettre des péchés pendant la prophétie, ils envisagent par là-même qu'ils soient de grands pécheurs (yafsiqū), mais le fisq ${ }^{14}$ des prophètes est minime et s'il se produit, leur attribut de grands pécheurs (fāsiq) se dissipe et s'annule (zāla l-ism 'an-hum), de sorte qu'ils ne soient pas visés dans les versets incriminant les grands pécheurs ${ }^{15}$. Ce propos est éclairé par un autre passage de l'ouvrage où Ibn Fūrak affirme que :

«Au sujet des péchés des prophètes, que le salut soit sur eux, on raconte qu'il [alAšarī] disait que les prophètes sont susceptibles de commettre des péchés mais qu'ils ne sont pas pour autant qualifiés de pécheurs (lā yusammūna bi-hā wa-lā yuqāl la-hum 'ușāt). Ceci n'est pas sa doctrine (mad̆habu-hu), mais celle de certains murği ites. Lui disait qu'on qualifie quelqu'un de pécheur à cause de l'occurrence de son péché, mais si [cette personne] se repent, la qualification de pécheur s'annule à cause de l'annulation de la chose signifiée ${ }^{16}$. "

Malgré le caractère indirect de cette source, le Muğarrad permet d'établir qu'al-Aš́arī s'est exprimé au sujet de la 'ịsma ; reste que la conception qu'il semble en avoir eu se situe bien loin de l'idée d'une préservation absolue du péché qui soit propre au prophétisme.

Cette tiédeur vis-à-vis d'une 'ișma étendue au domaine des mœurs se retrouve chez les disciples de la première génération. On apprendra par exemple du théologien juriste Abū Bakr al-Bāqillānī (m. 402/1013) que le fait de valider la possibilité de péchés commis par le Prophète Muhammad, en dehors de ce qui se rapporte à la transmission du message coranique, ne remet pas en cause sa sincérité et ne dispense pas de lui obéir ${ }^{17}$.

\footnotetext{
13. AL-Aš ARĪ, Muğarrad, p. 176.
}

14. Ce substantif désigne le statut juridique de « grand pécheur ».

15. Al-Ǎ̌́ ARĪ, Muğarrad, p. 158.

16. Al-Aš́ ARĪ, Muğarrad, p. 336.

17. AL-BĀQILLĀNī, al-Taqrīb, vol. 1, p. 438. Notons que cet auteur est souvent évoqué par les théologiens postérieurs comme un archétype de négation de l'impeccabilité des prophètes. À commencer par Ibn Hazm (m. 456/1064) qui se montre particulièrement critique à son endroit, comme l'indique cet extrait de son traité doxographique : «Quant à ce Bāqillānī, nous savons d'après l'ouvrage de son compagnon Abū Ǧa far al-Sumnānī, le cadi de Mossoul, qu'il prétendait que tout péché, qu'il soit infime ou énorme, est possible [de la part] des prophètes, sauf le mensonge dans la révélation. Il a admis qu'ils commettent de grands péchés en ajoutant que si le Prophète interdit quelque chose et qu'ensuite il se le permet, cela ne signifie pas qu'il y a eu abrogation, car il se peut qu'il le fasse en désobéissant à Dieu, et ce n'est pas à ses compagnons de le réprimander. Il a admis aussi qu'il puisse y avoir, dans la communauté de Muhammad, meilleur [homme] que lui, depuis son investiture jusqu'à sa mort », (voir IBN ḤAzm, al-Fiṣal, vol. 4, p. 2). 
Par ailleurs, il est évident que la notion de 'ịsma est étroitement liée à la dimension normative du prophète de l'islam, et c'est à travers la question de l'infaillibilité ou non de son effort de compréhension (iğtihād) qu'elle s'est posée aux théologiens juristes ašarites. L'étude d'Éric Chaumont sur l'uṣūlite šāfi ite et ašarite Abū Isḥāq al-Širāzī alFīrūzābādī (m.476/1084), qui illustre parfaitement ce lien entre la valeur de l'iğtihād de Muhammad et le débat sur la 'iṣma, nous informe qu'al-Širāzī n'avait pas admis une telle infaillibilité ${ }^{18}$. Et en poussant plus loin nos recherche sur l'opinion de cet auteur, notamment dans ses professions de foi, nous n'avons trouvé aucune allusion à toute autre dimension de la 'ișma ${ }^{19}$.

\section{AL-ĠAZĀLĪ (m. 505/1111) ET FAHुR AL-DĪN AL-RĀZĪ (m. 606/1209) : DEUX ATTITUDES OPPOSÉES}

L'argument suprême des premiers théologiens ašarites dans leur distinction entre un vrai prophète et un imposteur porte sur les miracles accomplis par Dieu en guise d'authentification, le Coran, ou Parole de Dieu révélée à Muhammad, étant considéré comme la preuve par excellence de sa véridicité.

Par ailleurs, le kalām mu'tazilite, où l'on retrouve l'origine de toutes les discussions développées par l'ašarisme, invoquait une autre catégorie de preuves pour justifier le prophétisme. Ces preuves sont liées à la personne même de Muhammad, et plus précisément, à sa véridicité (șidq), à son infaillibilité dans la transmission du message divin et à l'impeccabilité de ses mœurs ('ișma) ${ }^{20}$.

Cependant, en vertu du principe fondamental de la négation du bien et du mal rationnels (tahsin al-'aql wa-taqbihhihi) adopté par l'aš'arisme, si les hommes sont à même de saisir seuls les connaissances théoriques concernant leur milieu naturel, ils ne sont pas capables, sans l'aide d'une révélation divine, de distinguer le bien du mal en matière d'obligations religieuses ${ }^{21}$.

Si bien que l'évaluation du caractère moral du Prophète n'est possible qu'une fois que ce dernier a révélé la voie vertueuse à ses adeptes. Son impeccabilité, si toutefois elle était reconnue, ne pouvait donc pas constituer un argument pour prouver la véridicité d'un homme se disant prophète, puisqu'elle ne pouvait être saisie qu'a posteriori.

18. D'après É. Chaumont (1992, p. 130), « Šîrāzī emprunte à l'inverse de certains de ses condisciples, la voie la plus difficile : d'une part il tient que le Prophète était muğtahid exactement comme tout homme qualifié peut l'être, et d'autre part, il refuse, de manière cohérente, d'admettre l'idée que le Prophète soit infaillible. Si Šīrāzī ne reconnaît pas cette qualité au prophète, la thèse qu'il défend s'énonce pourtant ainsi : "il est possible que le Prophète (Dieu le bénisse et le salue !) soit dans l'erreur en son iğtihād. Néanmoins, il ne persévérait pas dans [l'erreur] mais s'en trouvait averti”.»

19. Notamment en ce qui concerne ses deux ouvrages 'Aqīdat al-salaf et al-Išāra ilā madhab ahl al-haqq. Certes la doctrine n'est pas niée explicitement, mais elle est éludée.

20. Voir GRIFFeL 2004, p. 101-102.

21. AL-ǦUWAYNī, al-Iršād, p. 258. 
La raison humaine n'étant pas apte, indépendamment de l'éthique enseignée par la religion, à juger de la qualité morale d'une personne ou d'un acte, dans ce système, les miracles sont restés pendant un temps le seul critère valable pour distinguer un véritable prophète d'un imposteur.

Par la suite, au sein d'un kalām ašarite plus tardif, dans lequel des éléments de philosophie avicennienne avaient été introduits, nous observons une évolution de la démonstration du prophétisme qui comptera dorénavant cette autre catégorie de preuves que sont les vertus morales ${ }^{22}$.

Nous abordons ici un aspect de ce que Louis Gardet avait contribué à identifier en distinguant «la voie des modernes », inaugurée par al-Ġazālì, de «la voie des anciens » au sein de la théologie ašarite ${ }^{23}$. Le critère le plus visible pour dater cette rupture semble avoir été l'introduction du syllogisme aristotélicien dans l'argumentation. Mais plus généralement, le phénomène qui a déterminé ce nouveau discours théologique est l'emprunt de doctrines à la falsafa. En dehors de ces apports extérieurs, il faut noter, comme c'est d'ailleurs le propre de tout progrès de la pensée humaine, que cette évolution fut portée par des courants internes à l'islam, et plus particulièrement au kalām. C'est ce qu'inspirent précisément des auteurs comme al-Ǧuwaynī (m.478/1085), l'un des plus illustres théologiens de la voie des "anciens », qui peut être considéré comme un précurseur de cette modernité dont il est question ici, tant son œuvre a engendré d'idées innovantes qui ont influencé les générations suivantes.

Dans le domaine de la prophétologie, cette évolution est marquée par l'introduction de critères nouveaux dans la démonstration de la validité du prophétisme de Muhammad, une démonstration dans laquelle l'invocation de la 'iṣma jouera un rôle capital.

En effet, comme nous allons le voir à travers les exemples d'al-Ġazālī puis de Fahr al-Dīn al-Rāzì, une connaissance préalable à ce que le prophète vient enseigner aux hommes sera dorénavant postulée, qui permet à l'être humain « d'apprécier » les qualités, notamment celle d'impeccabilité, d'un homme qui prétend être prophète.

Délaissant la classique démonstration par le miracle, fondée sur une perception sensible et prolongée par une transmission à chaînes multiples ininterrompues (al-tawātur), Abū Ḥāmid al-Ġazālī (m. 505/1111) a adopté une démonstration basée sur la saisie a posteriori des conséquences du prophétisme. À ce titre, ce penseur représente assurément une rupture dans la prophétologie sunnite ${ }^{24}$. Mais contrairement à ses successeurs, Il ignore

22. L'introduction d'éléments avicenniens dans la théologie aš'arite entre le début du xII ${ }^{\mathrm{e}}$ siècle et le début du xIII ${ }^{\mathrm{e}}$ est attestée entre autres par l'étude de F. Griffel qui souligne l'étendue de cette influence sur la prophétologie (GRIFFEL 2004, p. 103-114). Auparavant, B. Abrahamov avait fourni une illustration de ce mouvement à travers l'exemple de Faḩr al-Dīn al-Rāzī (ABRAhamov 1993, p. 415-425).

23. Cette distinction fait écho à ce qu'Ibn Haldūn avait identifié comme étant un tournant décisif dans les sciences du kalām (voir GARDET et ANAWATI 1948, p. 72-76).

24. Pour approfondir la distinction entre la preuve par le miracle, dite burhān al-inna (quia en latin) et la preuve par l'effet du prophétisme burhān al-limā (propter quid), voir GRIFFEL 2004, p. 109, n. 28. 
dans son credo la notion de 'ișma, laquelle n'apparaît que dans sa critique du chiisme ${ }^{25}$. Et lorsqu'il en parle dans ses réfutations du dogme de l'imām infaillible des bātinites, ce n'est visiblement pas pour en faire l'éloge :

«En ce qui concerne la 'ișma, elle n'est pas une caractéristique de la prophétie et l'affirmer ne prouve pas la prophétie. Certains groupes parmi nos condisciples ont soutenu que la 'ișma des prophètes concernant les petits péchés n'était pas démontrable. Ils ont pris pour exemple la parole de Dieu : "Adam désobéit à son seigneur et s'égara », ainsi qu'un ensemble d'histoires des prophètes $(. . .)^{26}$.»

"Le Prophète lui-même a dit: "Je juge sur les apparences; c'est Dieu qui a la charge des secrets", ce qui signifie : "Je juge d'après l'opinion générale recueillie auprès des témoins et il se peut que ces derniers se trompent". Les prophètes eux-mêmes n'ont aucun moyen de se prémunir de l'erreur dans ce genre d'effort de compréhension, alors comment lui [l'imām bāṭinite] peut-il espérer le faire ? ${ }^{27}$ »

Faḩr al-Dīn al-Rāzī n'empruntera pas la voie d'al-Ġazālī sur cette question. Certes, leurs prophétologies préférant une démonstration dite propter quid ${ }^{28}$ les mènent tous deux à centrer leur intérêt sur les qualités de Muhammad pour prouver la validité de son prophétisme, mais Ġazālī n'avait manifestement pas senti la nécessité de compter la 'îsma parmi ces qualités. Il en sera autrement pour son successeur qui adoptera cette doctrine en lui conférant une importance jusque-là inégalée dans le kalām ašarite.

En effet, al-Rāzī a consacré un traité intitulé 'ịsmat l-anbiyā' ${ }^{29}$ à la défense de l'impeccabilité des prophètes. Ce livre, qui a retenu notre attention, nous apprend en premier lieu que les divergences d'opinion de la communauté musulmane sur la ișma portent sur quatre points ${ }^{30}$ :

1 - Du point de vue de la croyance en Dieu (al-ítiqād), tous ces groupes, nous dit-il, ont affirmé l'impossibilité que les prophètes fassent preuve de mécréance ( $k u f r)$, excepté les fāạiliyya parmi les hāriğites. Ces derniers auraient affirmé que les prophètes ont commis des péchés, alors même que tout péché, selon eux, relève de la mécréance.

25. Nous avons en effet consulté deux œuvres fondamentales d'al-Ġazālī : Ihyyà ' 'ulūm al-dìn, ainsi que son unique ouvrage de kalām à strictement parler, Al-Iqtișād fï-li'tiqād, et aucun des deux ne mentionne la 'ișma.

26. AL-ĠAzĀLī, al-Mustaz̧hirī, p. 43.

27. AL-ĠazĀLĪ, al-Munqid, p. 90.

28. Voir supra, n. 24.

29. Dont l'essentiel est repris dans le Kitāb al-arba īn (p. 329-368) et, plus succinctement, dans al-Mahșūl (p. 501-502), du même auteur. Dans son ouvrage doxographique al-Fișal, Ibn Hazm traite de la 'isma sous une forme qui dépasse le simple exposé hérésiologique des premiers ašarites et s'impose comme un modèle chez Fahr al-Dīn al-Rāzī et plus tard al-Ĭği (m. 756/1355). La méthode consiste à donner une réfutation des fautes imputées par les adversaires présumés de la 'ișma à chaque prophète, suivant l'ordre chronologique de leur apparition. Ces accusations sont basées exclusivement sur des versets coraniques d'admonestation. Les plaidoyers pour disculper les prophètes sont de longueur variable et l'argumentation fait appel, selon les cas et les auteurs, à l'exégèse coranique, aux circonstances de la révélation (asbāb al-nuzūl), à l'examen de la validité des hadīt en relation avec celles-ci et à la philologie.

30. Voir AL-RĀZī, 'Ișma, p. 26-27. 
2 - Sur la question de la transmission des religions (tablïg al-šară’i i), il y a un accord unanime de la communauté sur l'impossibilité que les prophètes puissent commettre des altérations (tagiyir), pas même par inadvertance (sahw), sinon à remettre en cause la fiabilité des religions.

3 - Pour ce qui se rapporte aux décisions juridiques (fatwā), il est convenu ici aussi que les prophètes ne peuvent commettre une erreur. Mais un groupe aurait admis des erreurs par inadvertance.

4 - En ce qui concerne les mœurs (af äli-him wa-ạ̣wāli-him), Rāzī expose cinq points de vue :

a - Les littéralistes, qu'il appelle al-hašwiyya, affirment que les prophètes ont pu commettre de grands péchés intentionnellement (ta'ammudan).

b - Al-Ğubbāî̀ (m. 303/915-916) enseignait l'impossibilité que les prophètes commettent intentionnellement un péché, aussi petit soit-il, mais qu'ils pouvaient commettre des erreurs d'interprétation (hațầ' fïl-ta'wīl).

c - La majorité des mu'tazilites excluent les grands péchés mais ils conçoivent que les prophètes puissent commettre des fautes légères, excepté les actes répugnants.

d - Al-Naz̧āām (m. vers 230/845) soutenait l'impossibilité que les prophètes commettent de grands ou de petits péchés intentionnellement ou par erreur ; et la possibilité qu'ils fassent preuve d'oubli ou d'inadvertance, auquel cas ils sont réprimandés (yu'âtabūn) parce qu'ils se doivent d'exceller en matière de lucidité et de sincérité.

e - Les rāfidites affirment que les prophètes sont exempts de tout péché quel qu'il soit, et de tout oubli, inadvertance ou erreur de jugement et ce, de leur naissance à leur mort. Hormis cette école, écrit al-Rāzī, la communauté des musulmans considère que l'impeccabilité n'est vérifiée qu'après l'investiture des prophètes.

L'auteur fait enfin part de son opinion en affirmant qu'il est inadmissible que les prophètes commettent une faute, quelle qu'elle soit, si ce n'est pas inadvertance ou par oubli.

Pourtant, lorsqu'il entreprend d'expliquer les différents versets coraniques d'admonestation dont le sens apparent et les récits sur le contexte de la révélation (asbāb al-nuzūl) indiquent explicitement que le Prophète Muhammad a commis une faute, Rāzī se voit souvent contraint d'invoquer une justification qui ne figure pas clairement dans l'énoncé de son opinion, à savoir : l'erreur de jugement ou d'estimation (tark al-afḍl ou tark al-awlā) ${ }^{31}$.

31. Quant aux versets à portée générale qui ne concernent pas un événement particulier tels que Coran 48,2 : «Afin que Dieu te pardonne tes premiers et tes derniers péchés ( ... ) », de même que Coran 9, 117; 40, 55 ; 93, 7 et 94, 2, ils sont justifiés par des arguments rhétoriques et des versets contradictoires. Pour information, les autres versets invoqués par les négateurs de la 'ișma sont : Coran 5,$67 ; 6,52 ; 9,43 ; 10,94 ; 17,73-4 ; 33,1 ; 39,65$ et 87, 6. 
Ainsi, par exemple, au sujet du verset «Il s'est renfrogné et détourné car à lui est venu l'aveugle ${ }^{32} »$, notre auteur rapporte le récit de l'événement relatif à cette révélation en soulignant que le Prophète avait de quoi être gêné par l'intervention de cet "aveugle » alors qu'il conversait avec des notables de la tribu de Qurayš dans le but de les convertir. La réprimande divine qui s'en suivit porte donc, selon lui, sur une erreur d'estimation (tark alaf̣̂l) et en aucun cas sur un péché ${ }^{33}$. Les versets au sujet de la prise de rançon à la bataille de Badr («Il n'est d'aucun prophète de faire des captifs (...) $\left.{ }^{34} »\right)$ sont justifiés de la même manière.

Un autre événement de la biographie du Prophète invoqué par les opposants à la 'ịsma retient particulièrement l'attention d'al-Rāzī : il s'agit de la réticence de Muhammad à révéler son attirance pour Zaynab, l'épouse de Zayd, son fils adoptif. Les versets qui y sont relatifs, notamment: «(...) Quand tu craignais le jugement public alors qu'Allah est plus digne que tu le craignisses ${ }^{35}$ ! ", ne sont pas particulièrement blâmants, mais les récits rapportant cette histoire restituent un malaise que l'auteur tente de dissiper.

Sa propre justification est qu'Allah voulait abroger l'interdit affectant ce type d'alliance, mais, craignant les invectives des hypocrites, le Prophète se rétracta, alors qu'il eût été plus judicieux de divulguer son intention et de supporter les on-dit. Il n'est donc pas question ici non plus d'une quelconque faute et si le Prophète est sermonné, c'est uniquement pour ne pas avoir opté pour la meilleure solution (tark al-af̣al).

Quant aux récits relatant que Muhammad était secrètement amoureux de Zaynab, nous dit al-Rāzī, ils se fondent sur des informateurs uniques ( $\bar{a} h \bar{a} \bar{d})$, et il convient mieux de les rejeter afin de décharger le Prophète d'une telle chose. Mais il ajoute que si cela s'avérait juste, deux versions des faits sont rapportées : certains disent que dès lors que le Prophète avait vu et désiré Zaynab, elle était devenue proscrite pour Zayd.

L'auteur conteste fermement cette version, parce que si c'était vrai, en recommandant à Zayd de garder son épouse, d'une part, Muhammad aurait encouragé un adultère et, de l'autre, en qualifiant Zaynab d'épouse de Zayd, il aurait proféré un mensonge. "Or, ces deux faits, dit-il, sont indignes des musulmans et a fortiori du meilleur des prophètes ${ }^{36} . »$

D'autres ne soutiennent pas que Zaynab fut interdite à son époux, mais ils relatent qu'il devint obligatoire pour ce dernier de la répudier et que cela représentait une épreuve dans laquelle l'époux devait se conformer à la volonté de Dieu et de Son Prophète. Mais pour notre auteur, cette version est également outrageante pour le Prophète en ce qu'elle implique que si ce dernier ne maîtrisait pas son regard, il eut été possible qu'il s'arrêtât sur une créature près de laquelle il se fut alors trouvé, et étant donné que l'attirance est chose

32. Coran 80, 1-2.

33. Voir AL-RĀZī, 'Ișma, p. 137. Pour sa part, Ibn Ḥazm disculpe complètement le Prophète en affirmant que ce dernier a agi en croyant bien faire, parce que la conversion d'un homme influent qui risquait de lui échapper était prioritaire. Voir al-Fișal, t. 4, p. 22.

34. Coran 8, 67-68. Voir Al-RĀzī, 'Ișma, p. 132.

35. Coran 33, 37. Voir AL-RĀZī, 'Ișma, p. 129.

36. Al-RĀZī, 'Ișma, p. 131. 
involontaire (laysa bi-htiyāri-hi), il devenait nécessaire de contraindre l'époux à répudier sa conjointe. Partant, le Prophète avait le choix entre s'exposer aux diatribes ou enfreindre la Révélation s'il n'imposait pas le divorce. Mais l'auteur de conclure qu'afin d'éviter ces deux grands périls, Muhammad se surpassait en évitant les regards (kāna yubālig fí-hifž alnazar) ${ }^{37}$.

L'épisode des versets sataniques est un autre événement édifiant de la vie du Prophète qui offre un argument de choix aux adversaires de la 'iṣma. Bien plus que l'impeccabilité, c'est l'infaillibilité dans la transmission de la révélation qui est ici en jeu.

La révélation coranique ayant informé le Prophète que: "Avant toi, Nous n'avons envoyé nul apôtre et nul prophète, sans que le démon jetât l'impureté dans leur souhait (tamannī) lorsqu'ils le formulaient. Allah abrogera donc ce que le démon jette [d'impur en ton message] ${ }^{38}$, les négateurs de la 'ișma considèrent que le sens apparent de ce verset indique que Satan répand de quoi semer le doute sur la récitation des prophètes. Al-Rāzī s'oppose d'emblée à cette lecture parce qu'elle annule toute confiance en la validité de la révélation. Il rapporte le récit relatif à cette affaire en citant le passage qui fut supprimé après les versets 19-20 de la sourate 53 (al-Nağm) : «Ce sont les sublimes Déesses et leur intercession est certes souhaitée » et glose les justifications de ses prédécesseurs ${ }^{39}$.

Le mot «al-tamannī» revêt deux sens, nous dit-il, le premier est le souhait qui émane du cœur et le second est la récitation. Certains exégètes ont opté pour la première signification que l'auteur juge faible en se fondant sur la suite de la sourate 22, indiquant que l'impureté répandue par Satan sert de tentation aux injustes. Or, si cette tentation était abrogée au stade de souhait, soit avant d'être récitée par le Prophète, elle ne pourrait être mise à contribution.

L'auteur en vient à dire qu'il s'agit donc de récitation et entame une discussion dans laquelle il s'oppose radicalement à l'idée que Muhammad ait pu prononcer le verset proscrit, pour s'aligner sur ceux qui prétendent que Satan avait mêlé sa voix à celle du Prophète.

Les tenants d'une récitation par le Prophète prétendent pour certains que, contrairement à ce qui a été compris par les infidèles en présence, le ton de la phrase « et leur intercession est certes souhaitée » se voulait interro-négatif (istifhām inkār), l'intention étant de nier un quelconque pouvoir d'intercession chez les idoles en question, alors que d'autres invoquent un moment d'inadvertance ( $s a h w)$ dans la récitation.

Rāzī exclut les deux thèses, la première parce que la phrase proscrite s'accorde tant au niveau du sens que du rythme avec le reste de la sourate et induit les auditeurs à croire qu'elle est laudative, ce qui revient au même ; la seconde parce qu'il est peu probable que soit produit un tel accord de sens et de rythme dans un moment d'inadvertance, sans compter

37. AL-RĀzī, 'Ișma, p. 132.

38. Coran 22, 51-52 ; AL-RĀZ̃̄, 'Ișma, p. 121-122.

39. Ibid. Pour connaître l'opinion d'al-Rāzī sur cette affaire il convient de se reporter à son exégèse coranique alTafsìr al-kabìr dans lequel il n'accorde aucun crédit aux récits rapportant cet épisode et nie par là-même la relation de cause à effet entre le verset proscrit et Coran 22, 51-52 (voir AL-Rāzī, al-Tafsìr, vol. 23, p. 44). 
qu'admettre une telle absence dans la récitation du Coran remet en cause la fiabilité de la révélation. Cet argument est réutilisé par notre auteur contre ceux qui prétendent que Satan a contraint le Prophète à formuler la phrase interdite ${ }^{40}$.

Al-Rāzī admet néanmoins la possibilité de trois autres explications. La première affirme que c'est Satan qui a récité le verset en question. Ce serait donc sa voix et non celle du Prophète qui aurait été entendue par l'assistance ; et al-Rāzī d'ajouter que ce n'est pas impossible, d'autant que cela disculpe complètement le Prophète. Quant à objecter que cela peut remettre en cause la fiabilité de la révélation, il répond qu'en vertu de Sa sagesse (hikma), Dieu aurait nécessairement déjoué toute autres tromperie sataniques afin que l'intégrité de la révélation fût préservée ${ }^{41}$. La seconde explication prétend que ce sont les qurayšites présents à ce moment-là qui ont ajouté la phrase laudative concernant leurs idoles. Sachant que le Prophète avait cité leurs noms dans l'intention de les insulter, ces derniers se seraient empressés de rajouter cette phrase dans le but de le confondre. L'ultime thèse agréée par notre auteur énonce que le mot «al-Ǵarāniqq» (les grues) renvoyait en réalité à des anges ; seulement lorsque les associationnistes ont imaginé qu'il s'agissait de leurs déesses, Allah a abrogé ce passage ${ }^{42}$.

De par ses justifications visiblement plus dialectiques que scripturaires, al-Rāzī établit une 'ișma dont la conception est très proche de celles des mu'tazilites ${ }^{43}$. Hormis des erreurs d'estimation ou d'inadvertance immédiatement corrigées par le Prophète lui-même, il rejette l'idée qu'aucune autre forme de faute puisse lui être attribuée durant sa mission. C'est précisément cette conception de l'impeccabilité des prophètes qui sera défendue plus tard par les théologiens ašarites à l'instar d'al-İ̆ḡi (m. 756/1355) ${ }^{44}$.

\section{LA NOTION DE 'IȘMA CHEZ IBN TAYMIYYA}

Quelques décennies plus tard, au détour d'un essai de définition du terme nabī, (messager ou informateur), le théologien juriste Ibn Taymiyya (m. 728/1328) note que la caractéristique de cette sorte de messager que sont les prophètes est d'être véridiques dans la transmission du message divin:

40. Voir AL-RĀZī, 'Ișma, p. 126.

41. Notons que l'argument de la sagesse divine protectrice aurait pu être brandi pour justifier les cas où Muhammad aurait récité ce verset, que ce soit par inadvertance ou par influence satanique. Mais al-Rāzī semble bien déterminé à évacuer l'idée d'une possible altération de la révélation par le Prophète, fut-elle corrigée immédiatement par Dieu.

42. Al-R̄̄ZĪ' 'Ișma, p. 127.

43. La source mu'tazilite la plus exhaustive sur cette question est sans nul doute le Muġnī du cadi 'Abd al-Ǧabbār (m. 415/1025). La discussion sur le critère de répugnance (tanfï) discriminant les petites fautes envisageables de toutes celles qui sont inadmissibles, de même que la démonstration de cette inadmissibilité, y sont purement intellectuelles, les arguments coraniques étant remarquablement absent, voir al-Mugin̄i, vol. 15, p. 281-304.

44. Pour İ̆̄i les récits sur les péchés des prophètes inspirés du Coran, du Hadith ou des traditions, sont systématiquement réfutés lorsqu'ils émanent d'informateurs uniques. Pour le reste, l'auteur préconise de dégager d'autres interprétations, et si ce n'est pas possible, il convient de considérer que cela porte uniquement sur la période qui précède leur mission, sinon il s'agit nécessairement d'omissions vénielles (șaḡảir șadarat sahwan) ou

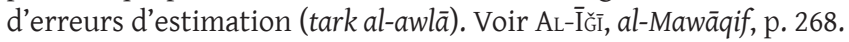


«Le messager (nabī) est celui qui transmet ce que Dieu lui a révélé sans y inclure aucun mensonge ou contradiction intentionnelle ('amdan) ou involontaire (hata'). Voilà ce que signifie le terme infaillibles (ma'șūmūn) en ce qu'ils transmettent de Dieu ${ }^{45}$. »

Sans doute conscient des multiples portées attribuées à la notion de ișma, il ajoute aussitôt que le Coran parle plus explicitement de véridicité (sidq) lorsqu'il s'agit de qualifier le Prophète. Et c'est, selon lui, précisément cette véridicité qui est l'objet des signes (al$\bar{a} y \bar{a} t)$ et des démonstrations (al-barāhìn) contenues dans le Coran :

"Quant au terme 'iṣma, il est énoncé dans le Coran à : « Et Dieu te protégera des gens » (wa-allāhya ș̣imu-ka min al-nās ), c'est-à-dire de leur malveillance. Le sens de ce mot [ma'șūm] dans le Coran est: «celui que Dieu protège du mensonge volontaire ou involontaire ». Et l'utilisation de termes coraniques pour définir les vérités du dogme est préférable à l'utilisation d'autres termes. (...) Aussi le Prophète est-il [dit] ma'șūm selon la langue du Coran. C'est-à-dire que Dieu le protège des démons (al-šayāțīn), ceux des humains et des djinns. Et qu'Il les défend d'altérer le message qu'il transmet ou bien d'empêcher sa transmission de sorte que le Prophète ne dissimule jamais et ne mente jamais ${ }^{46}$. »

Sans s'inscrire totalement en faux contre le pragmatisme des disciples d'Ibn Hanbal, qui, rappelons-le, rejettent la 'ịma en s'appuyant sur des preuves scripturaires, Ibn Taymiyya limite la portée de la 'iṣma à l'infaillibilité du Prophète dans la transmission du message coranique.

Notons que parmi les adversaires de choix de ce polémiste figurent les rāfidites qui prônent l'impeccabilité de leurs imāms, et les chrétiens auxquels il reproche leurs excès dans l'exaltation de la personne de Jésus. Autant d'éléments qui sont déterminants dans la formulation de son opinion. Pour illustrer ce propos, nous analysons une dissertation qui figure dans son Minhāğ al-sunna ${ }^{47}$ et dont le ton est particulièrement polémique, puisqu'il s'agit de réfuter la 'iṣma telle que conçue par les rāfị̣ites.

En s'appuyant sur le traité d'hérésiographie d'al-Ašarī (Maqālāt al-islāmiyyīn), Ibn Taymiyya commence par objecter que l'opinion des rāfiḍites sur la 'ișma n'est pas homogène puisque certains d'entre eux, tels Hišām b. al-Ḥakam (m. 179/ 795), vont jusqu'à considérer que les prophètes sont susceptibles de commettre des péchés qu'une révélation vient corriger, à l'inverse des imāms qui sont absolument impeccables puisqu'ils ne bénéficient pas de révélations. Il énonce ensuite ce qui semble être l'unique sens qu'il attribue à cette notion, à savoir l'infaillibilité dans la transmission du message divin :

«Deuxièmement, il est donné que les musulmans sont d'accord sur l'infaillibilité [des prophètes] en ce qu'ils transmettent de Dieu, [car] cela garantit l'objectif de la mission [prophétique] ${ }^{48}$.»

45. IBn TAYMiYYa, al-Nubuwwāt, p. 355.

46. IBn TAYMiYYa, al-Nubuwwāt, p. 355-356.

47. Voir IBN TAYMIYYA, Minhāăğ, p. 226-228. Cette œuvre majeure est une réfutation systématique du Minhāă al-karāma du théologien al-Hillī (m. 725/1325).

48. Iвn TAYmiYya, Minhāğ, p. 226. 
Suit un passage pivot qui ne manque pas d'ironie, à partir duquel l'auteur aborde la critique des tenants d'une impeccabilité du Prophète semble-t-il abusive à son goût. Dénonçant les conséquences d'une telle 'ișma qui signifie être à l'abri de toute erreur, il tente de démontrer que cette conception de ses adversaire chiites porte préjudice aux prophètes:

«Aussi, il faudrait [donc] que le Prophète n'ait pas à se repentir pour gagner l'amour de Dieu et sa joie, en vue d'élever son rang et qu'en [conséquence de] ce repentir désiré par Dieu, il devienne meilleur qu'avant.

Outre les contradictions [que présente] cette opinion avec le Livre et la sunna, il s'agit d'une atteinte à la dignité des prophètes et d'un reniement de leur rang, excluant la charité de Dieu envers eux, de même que sa miséricorde et son pardon. Celui qui va croire que toute personne n'ayant jamais péché ni même été infidèle est meilleure que quiconque a cru après avoir été impie ou bien s'est repenti après avoir péché va à l'encontre de ce qui est clairement enseigné par l'islam. Il est évident que les compagnons qui ont cru le Prophète après avoir été incrédules et qui se sont repentis de leurs péchés, Dieu les ayant guidés après leur errance, sont plus vertueux que leurs enfants qui sont nés dans l'islam. On ne peut confondre les enfants des anșār ${ }^{49}$ avec les anșār et les enfants des muhāğirūun ${ }^{50}$ avec les muhāğirūn que si l'on est ignorant. Mais où [se situe donc] l'homme qui se transporte du péché vers la vertu par la réflexion (nazar), la démonstration (istidlāl), la patience (șabr) et son effort de compréhension (iğtihād), [ajoutant à cela] l'abandon de ses habitudes et le reniement de ses amis, etc. par rapport à celui qui n'a rien connu de tel ? ${ }^{51}$ »

Aussi, contre l'argument répandu parmi des théologiens de toutes tendances, et qu'il attribue ici à al-Hillī (m. 725/1325), selon lequel un péché commis par un prophète induirait une répugnance (yūğỉb al-tanfîr) et entamerait son intégrité, Ibn Taymiyya est formel : une telle intégrité n'est absolument pas altérée par des péchés expiés. Bien plus, soustraire cette catégorie d'êtres que sont les prophètes au besoin de miséricorde divine procède de l'orgueil. Et comme il se doit pour un partisan de la supériorité des preuves scripturaires, l'auteur ne prive pas son argumentation de traditions où le Prophète lui-même se décrit comme n'étant pas à l'abri de l'erreur, traditions parmi lesquelles on retrouve l'expression du spectre de la divinisation de Jésus qui est manifestement l'une des causes principales de la défiance d'Ibn Taymiyya envers cette doctrine :

«Il est attesté dans le Șahịh que le Prophète a annoncé : "Personne d'entre vous n'entrera au paradis par son action; on lui répondit : pas même toi, ô envoyé de Dieu? Pas même moi, sauf si Dieu étend sa miséricorde et sa faveur sur moi." C'était l'un de ses sermons les plus fameux. Aussi, que Dieu le bénisse et le salue, dit-il : "Ne m'exaltez pas comme les chrétiens ont exalté Jésus, le fils de Marie ; au demeurant, je suis un homme, alors dites que je suis le sujet de Dieu et son envoyé." Tous ceux qui l'ont entendu l'ont loué pour ces paroles. Les deux Șahịh relatent qu'il récitait aussi : "Mon Dieu, pardonne-

\footnotetext{
49. Ainsi sont désignés les habitants de Médine, ville où le Prophète s'exila avec ses compagnons, qui se convertirent à l'islam.

50. Les exilés sont les compagnons de la première heure qui ont suivi le Prophète Muhammad dans son exil vers Médine.
}

51. IBN TAYMiYYa, Minhāğ, p. 226. 
moi mon erreur, mon ignorance, mes excès et ce que Toi seul sais de moi. Mon Dieu, pardonne-moi la facétie, l'imprévisibilité, l'erreur et son intention, et toutes ces choses en moi. Mon Dieu, pardonne-moi mes premières et mes dernières [erreurs], celles que j'ai dissimulées et celles que j'ai divulguées ainsi que ce que Toi seul sais de moi. Tu es celui qui anticipe (al-muqaddim) et celui qui ajourne (al-mu'ahhir), tu es l'omnipotent ${ }^{52}$. »

L'ultime argument développé par notre auteur ne manque pas d'originalité et porte sur l'édification de l'unicité divine (al-tawhīd) ${ }^{53}$. Pour Ibn Taymiyya, une 'iṣma qui impliquerait l'absence du besoin de miséricorde divine dépasserait les limites de Dieu, ou plus exactement, celles de la seigneurialité (al-rubūbiyya). En effet, l'atteinte à l'unicité de Dieu est l'objet d'une doctrine remarquablement sophistiquée chez cet auteur. Pour lui, personne n'échappe à ce travers, fût-ce sous des formes quasi imperceptibles. Il n'est donc pas surprenant que, de son point de vue, la 'iṣma des prophètes prêchée par les chiites engendre l'associationnisme (al-širk) ${ }^{54}$ :

«L'absence de besoin est une caractéristique de la seigneurialité (al-rubūbiyya). Quant à l'être humain, son accomplissement (kamāl) réside dans son besoin de Dieu, dans la dévotion, la pauvreté et l'indigence. Plus sa dévotion s'accomplit, plus il s'améliore. Et l'occurrence de ce qui l'astreint au repentir (șudūr mā yuḥigiğu-hu ilā al-tawba) et au pardon contribue à augmenter sa dévotion, son dénuement et son humilité ${ }^{55}$. »

Ibn Taymiyya affronte ses adversaires au sujet de la 'ịma dans un autre passage du Minhā $\check{g}^{56}$. Il s'agit cette fois-ci de démentir, dans une dissertation plus courte, une allégation portée par les rāfidites contre les sunnites qui, selon leurs détracteurs, considéreraient que les prophètes ne sont pas impeccables (g்ayr ma'șūminn). L'auteur reformule la position consensuelle des sunnites sur l'infaillibilité du Prophète dans la transmission de la révélation et ajoute qu'aucune erreur ne saurait persister dans ce domaine. La controverse des versets sataniques est évoquée à ce propos et Ibn Taymiyya ne nie pas l'éventualité d'une inspiration satanique qui aurait toujours été rectifiée par Dieu. Aussi la possibilité de commettre des péchés n'est-elle pas rejetée, mais les prophètes ne persisteraient pas en tout ce qui remet en cause leur statut de messager. L'éloge du repentir réapparaît brièvement, puis ce sont les thèmes de l'omission et de l'inadvertance qui sont développés. L'auteur reconnaît non seulement que les prophètes ont pu faire preuve d'inadvertance mais que ce faisant, ces derniers fondaient la norme. Une phrase de Muhammad rapportée dans le Muwațta' de l'imām Mālik est donnée en exemple : « [Il arrive] que j'omette et j'omets afin [d'établir] la tradition (sunna)». Ibn Taymiyya développe enfin le sujet de l'excès (al-g̈uluw)

52. IBN TAYMiYYA, Minhāăg, p. 227.

53. Voir Abrahamov 1993, p. 24-25.

54. Ibn Taymiyya a complexifié l'idée d'unicité divine en distinguant au moins trois paradigmes : la seigneurialité (al-rubūbiyya), la divinité (al-ulūhiyya) et les noms et les attributs (al-asmā' wa-l-șifāt). Voir Мıснт 2002.

55. IBN TAYMIYYA, Minhāă, p. 228.

56. IBN TAYMIYYA, Minhāăg, p. 130. 
des rāfidites dans la glorification des prophètes et des imāms, un excès qui, selon lui, les a menés, comme ce fut le cas des chrétiens, à l'associationnisme ${ }^{57}$.

\section{CONCLUSION}

Dans la continuité de leur maître al-Ašcarī, dont la volonté était de concilier la tendance des ahl al-hadị (les partisans des traditions prophétiques) avec la dogmatique spéculative, les premiers ašarites avaient adopté la 'ișma des prophètes en se gardant d'une conception qui remettait en cause le sens littéral des versets d'admonestation. L'édification du miracle coranique (ǐğāz al-qur'ān) semble avoir été leur seule ambition lorsqu'ils proclamaient l'infaillibilité du prophète dans la transmission de la révélation.

Quelques siècles plus tard, Ibn Taymiyya fit montre du même discernement vis-à-vis de cette doctrine. Certes, comme nous l'avons vu, son discours sur la question ne manque pas d'être original, mais au final, son opinion rejoint ce qui était enseigné jadis par les anciens de l'aš́arisme. Il y a évidemment une part de réaction au christianisme et à la prophétologie chiite dans son discours, mais à l'inverse des aš́arites tardifs, il se démarque clairement du rationalisme chiite et mu tazilite dans le traitement de cette question ${ }^{58}$.

En somme, nous retiendrons que le parcours de la doctrine de l'impeccabilité des prophètes au sein du sunnisme est marqué par un paradoxe. C'est une idée qui ne peut être vérifiée par un raisonnement démonstratif ou validée par les informations recueillies par voie de transmissions multiples et ininterrompues (tawātur). Il s'agit tout au plus d'un postulat associé à celui d'un Dieu nécessairement bienveillant pour l'humanité, adopté par les courants les plus favorables à la primauté de la raison dans l'inter prétation de la révélation de Muhammad, ceux que les historiens de la pensée appellent « les rationalistes » ${ }^{59}$; alors même que les littéralistes qui rejetaient cette primauté de la raison, se référant au sens obvie des sources scripturaires que sont le Coran et la tradition prophétique, s'étaient érigés contre cette doctrine. Et il fallut attendre la synthèse d'un Ibn Taymiyya pour voir ce rejet justifié par des arguments dialectiques.

57. IBn TAYMIYYA, Minhāğ, p. 130.

58. Dans son éminente thèse sur la pensée d'Ibn Taymiyya, Henri Laoust s'était trompé en attribuant à son auteur une opinion sur la 'ịsma calquée sur celle d'al-Hillī : «C'est surtout cependant à sa prophétologie, formée en communion d'idées assez étroite avec le shiisme, qu'Ibn Taymiyya a recours : il voit, dans l'infaillibilité et la perfection de Muhammad, la certitude que suivre la Sunna revient à suivre le Coran » (LAoust 1939, p. 295). En se fiant exclusivement à cette étude pour illustrer l'opinion d'Ibn Taymiyya, Louis Gardet avait reproduit la même erreur (GARDET 1967, p. 186).

59. Sur ce point, nous rejoignons l'avis de J. Van Ess selon lequel « au contraire c'était eux, les mu'tazilites, qui au moment du plus grand effort de leur rationalisme, donnèrent des impulsions décisives à la glorification du Prophète, et par cela contribuèrent sans en douter, à la victoire du hadīt » (voir VAN Ess 1982, p. 222). 


\section{RÉFÉRENCES}

\section{Sources}

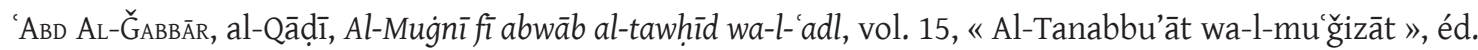
M.M. Qāsim [et al.], Le Caire, Al-dār al-mișriyya li-l-ta'līf wa-l-tarğama,1961-65.

Aš ARĩ (AL-), Abū al-Ḥasan, Maqālāt al-islāmiyyīn wa-hntilāf al-mușallīn, 2 vol., Le Caire, Maktabat al-nahḍa al-mișriyya, 1950.

Aš ARİ (AL-), Abū al-Ḥasan, al-Ibāna 'an ușūl al-diyāna, Le Caire, al-Ğamal, 1928.

Aš́ARĩ (At-), Abū al-Ḥasan, Kitāb al-luma' fì al-radd 'alā ahl al-zayg் wa l-bida', Beyrouth, Imprimerie Catholique, 1953.

Aš ARĪ (AL-), Abū al-Ḥasan, Muğarrad Maqālāt al-Aš arī, éd. D. Gimaret, Beyrouth, Dār al-Mašriq, 1987.

BAĠdĀDī (AL-), Abu Manșūr, Ușūl al-dīn, Istanbul, Madrasat al-ilāhiyāt bi-dār al-funūn al-turkiyya,1928.

BAQILlĀNī (AL-), Abū Bakr, al-Taqrīb wa-l-iršād, vol. I, Beyrouth, al-Risāla, 1957.

Coran, trad. Régis Blachère, Paris, Besson-Chantemerle, 1998.

ĠAZ̄̄LI (AL-), Abū Ḥāmid, al-Munqị̂ min al-ḍalāl, trad. F. Jabre, Beyrouth, Librairie orientale,1969.

ĠAzĀLī (AL-), Abū Ḥāmid, Ihyyā' 'ulūm al-dīn, vol. 2, Le Caire, 'Īsā al-Babī al-Ḥalabī wa-awladuhu, 1933.

ĠazĀLì (AL-), Abū Ḥāmid, al-Mustazhirī (Streitschrift des Ghazâlî gegen die Batiniyya-Sekte), éd. I. Goldziher, Leiden, Brill, 1956.

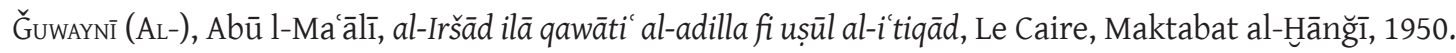
Iвn BATṬA, al-Šarh wa al-ibāna 'alā uṣūl al-sunna wa-l-diyāna, trad. H. Laoust, Damas, Institut français de Damas, 1958.

IBN FūRAK, Abū Bakr, Muğarrad maqālāt al-Ašcarī, éd. D. Gimaret, Beyrouth, Dār al-Mašriq, 1987.

Iвn ḤAzм, Abū Muhammad Alī, Kitāb al-Fișal fì l-milal wa-l-ahwā’ wa-l-nihal, Bagdad, Maktabat al-Mutannā, 1970.

IвN Hı̌̌̄̄m, Sirrat al-nabī, Le Caire, Dār al-tahrīir li-l-țab` wa-l-našr, 1989.

IвN MAnž̄r, Lisān al-arab, vol. 10, Beyrouth, Dār Șādir, 2000.

Iвn TAymiYYA, Taqī al-Dīn Ahmmad, Minhāğ al-sunna al-nabawiyya fì naqḍ kalām al-šř a wa-l-qadariyya, Le Caire, Bulaq, 1903.

IвN TAYмiYYA, Taqī al-Dīn Aḥmad, al-Nubuwwāt, Beyrouth, Dār al-qalam, 1985.

İĞ̄i (AL-), 'Aḍud al-Dīn, al-Mawāqif fí 'ilm al-kalām, Beyrouth, Dār al-Ǧìl, 1997.

RĀzī (AL-), Faḩr al-Dīn, al-Maḥsūl fíi ilm al-ușūl, vol. 1, Beyrouth, Dār al-kutub al-iilmiyya, 1988.

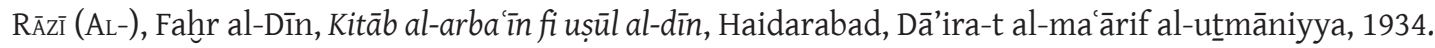

RĀzĩ (AL-), Faḩr al-Dīn, 'Ișmat al-anbiyā', Beyrouth, Dār al-kutub al-'ilmiyya, 1988.

RĀZī (AL-), Faḩr al-Dīn, al-Tafsīr al-kabīr, 16 vol., Beyrouth, Dār al-kutub al-'ilmiyya, 2000.

ŠìrĀZī (AL-), Abū Ishāā, 'Aqīdat al-salaf, éd. Marie BernAnd, Le Caire, IFAO, 1987.

ŠĩrāZī (AL-), Abū Isḥāq, al-Išāra ilā madhab ahl al-ḥaqq, éd. Marie Bernand, Le Caire, IFAO, 1987.

WENSINCK, Arent Jan, Concordance et indices de la tradition musulmane, Leyde, Brill, 1938.

Études

Aвrahamov, Benyamin, 1993 : «Ibn Taymiyyah and the doctrine of 'ișmah », The Bulletin of the Henry Martyn Institute of Islamic Studies 12/3-4, p. 24-25. 
Aвrahamov, Benyamin, 2000 : "Religion versus philosophy : The case of Fahr al-Dīn al-Rāzìs proofs of prophecy", Oriente Moderno 19, p. 415-425.

Chaumont, Éric, 1992 : «La problématique classique de l'Iğtihād et la question de l'Iğtihād du prophète : Iğtihād, wahy et 'ișma », Studia Islamica 75, p. 105-139.

GARDET, Louis et ANAWATI, M. M., 1948 : Introduction à la théologie musulmane, Paris, Vrin, 1948.

GARDET, Louis, 1967 : Dieu et la destinée de l'homme, Paris, Vrin.

Gimaret, Daniel, 1990 : La doctrine d'al-Aš arī, Paris, Cerf.

GrifFeL, Franck, 2004 : "Al-Ġazālī's Concept of Prophecy : The Introduction of Avicennan Psychology into Aš'arite Theology", Arabic Sciences and Philosophy 14, p. 101-144.

Laoust, Henri, 1939 : Essai sur les doctrines sociales et politiques de Taqi-d-dīn Ahmad b. Taymiyya, canoniste hanbalite né à Harrān en 661/1262, mort à Damas en 728/1328, Le Caire, IFAO.

LAoust, Henri, 1979 : Les Schismes dans l'Islam, Alger, SNED.

Madelung,Wilferd, 1971 : « 'ișma », Encyclopédie de l'Islam (2éd.), Leiden, Brill, vol. IV, p. 190-92.

Мıснот, Yahya, 2002 : «Entre la divinité et la seigneurialité, le polymorphisme de l'associationnisme

(širk)», Textes spirituels d'Ibn Taymiyya, <http://www.muslimphilosophy.com/it/works/ITA\%20 Texspi\%2004.pdf>.

SFAR, Mondher, 1998 : Le Coran, la Bible et l'Orient ancien, Paris, Sfar.

VAN Ess, Josef, 1982 : «L'autorité de la tradition prophétique dans la théologie mu'tazilite », MAKDISI, Georges, Sourdel, Dominique et Sourdel-Thomine, Jeanine (éd.), La notion d'autorité au Moyen Âge, Islam, Byzance, Occident, Paris, PUF, p. 211-226.

Wensinck, Arent Jan, 1965 : The Muslim Creed, Londres, Frank Cass \& Co. Ltd ( $2^{\mathrm{e} e}$ éd.). 